\title{
A Novel Surgical Technique of Removal of Sub-Macular Hemorrhage in Post Traumatic Cases
}

\section{Mohammad Khalife ${ }^{1 *}$, Mojtaba Abrishami ${ }^{2 *}$, Purva Date $^{3}$ and Matteo Forlini $^{4}$}

${ }^{1}$ IC Center, Beirut, Lebanon

${ }^{2}$ Khatam-al-Anbia Eye Hospital, Eye Research Center, Mashhad University of Medical Sciences, Mashhad, Iran

${ }^{3}$ Valvekar Medical and Research Centre, Solapur, India

${ }^{4}$ Department of Ophthalmology, San Marino State Hospital, San Marino

*Corresponding Author: Mohammad Khalife, IC Center, Beirut, Lebanon and

Khatam-al-Anbia Eye Hospital, Eye Research Center, Mashhad University of Medical

Sciences, Mashhad, Iran.
Received: March 08, 2021

Published: March 18, 2021

(C) All rights are reserved by Mohammad

Khalife., et al.

\begin{abstract}
Purpose: To describe a new technique in removing sub-macular hemorrhages (SMH) in traumatic patients and results.

Methods: In this technique, patients with SMH, a complete three port vitrectomy was performed. Using a back flush instrument and soft tip needle, considering safe distance from silicon tip to retina, SMH is swept using gentle flushes to an other site far from the macula in which clots were removed via a retinotomy site. In a case with macular hole, the hemorrhage was drained via the hole.

Results: In case one, SMH was swept to the temporal of the macula and removed via a retinotomy. Best corrected visual acuity was improved from hand motion to 20/ 30. In the second patient, four weeks after trauma, SMH was removed and after three weeks the BCVA was improved to 20/25 (after three weeks). In the third case that had traumatic macular hole in first place, the SMH was drained via the macular hole and BCVA was improved from hand motion to 20/100.

Conclusion: Sub-Macular hemorrhage sweeping using back flush after a complete vitrectomy seems to be efficacious in restoring macular anatomical and visual function.
\end{abstract}

Keywords: Sub Macular Hemorrhage; Vitrectomy; Visual Function; Trauma

\section{Introduction}

Ocular trauma may induce choroidal rupture and as choroid is a bloodful tissue, it usually result in submacular hemorrhage (SMH) [1]. Subretinal hemorrhage damages tissue through a variety of mechanisms: the presence of iron hemosiderin and fibrin in the blood has toxic effects on the overlying photoreceptors, mechanical effect due to clot retraction can sheer and damage the photoreceptors, and physical separation of the photoreceptors from the retinal pigment epithelium (RPE) causes both atrophy and can end with disciform scar formation. Because of hematotoxicity and hemosiderin effects on the retina, the outcome for untreated SMH is poor and subsequently fibrotic degeneration of the retina and RPE may worsen the condition. In some cases, choroidal neovascularization may happen [2,3].
In order to recover vision, in patients with $\mathrm{SMH}$, a surgical procedure is proposed, usually with the intravitreal injection of gas only or gas with recombinant tissue plasminogen activator ( $t-P A)$ without carrying out a vitrectomy [4-7]. Moreover, vitrectomy, subretinal injection of t-PA and air tamponade was performed with good visual outcome. However, in these technics, SMH is not removed and just helped in displacing the hemorrhage, from the macula to anywhere else.

Here, we aim to report a novel surgical technique to remove and drain SMH via a retinotomy site AWAY the macula, after a deep three port pars plana vitrectomy. 


\section{Methods}

\section{Patients}

In an interventional case series, conducted at Beirut Eye and ENT (Ear, Nose, Throat) Specialist Hospital - BESH, Beirut, Lebanon, patients with traumatic submacular hemorrhage and visual reduction whom their diagnosis was confirmed by Optical Coherence Tomography (TOPCON) were included. Patients had a history of blunt trauma and open globe injuries were excluded. Best corrected visual acuity and refraction was also acquired. The study and its data acquisitions all were approved by the Ethical Committee of Beirut eye and ear specialist hospital. Patients were enrolled after providing written informed consent, in compliance with the Declaration of Helsinki. Written informed consents for publication of the clinical details and clinical images were obtained from the patients.

\section{Surgical method}

In all patients standard three port 23 gauge triamcinolone acetonide assisted pars plana vitrectomy was performed and posterior vitreous detachment was induced. Because of traumatic insult, periphery of the retina was checked for any retinal break, and in case, barrier laser was done. After that, using a back flush and a 23 gauge silicone soft tip needle, subretinal hemorrhage were swept from the temporal of the disc to a retinotomy site away of the macula. The distance from the soft tip to the retina was set to be secure, not so near to traumatize the retina or not so far to reduce the efficacy of liquid flush, usually 2 - 3 millimeters. Using intraocular diathermy, a retinotomy site was performed at least one disc diameter far from the macula. By applying pressure on the silicon shaft of the back flush, shock wave will allow the big submacular clot to break allowing us to sweep the smaller clot through the retinotomy site and so on for the rest of clots until hemorrhage was totally drained. The retinotomy site was barried by light endolaser spots. No active or passive draining through retinotomy site was done. In case of macular hole, the clots were been pushed via the macular hole. After air/fluid exchange, SF6 or silicone oil WERE used, based on the other effects of the trauma on the eye.

One week, one month and three months after surgery, patients were followed and OCTs were performed for the patients.
Case 1

A 22 year old male patient with a history of car accident and head trauma ten days before was referred. In fundus exam and OCT, choroidal ruptures not involving the macular center and submacular hemorrhage extended to the superotemporal of the macula with visual acuity of hand motion in the left eye were found. Due to macular involvement, pars plana vitrectomy with submacular hemorrhage sweeping and drainage from a retinotomy near the superotemporal retinal vein was performed. Endolaser around the retinotomy was done and eye was filled with SF6 gas. After 4 weeks, final visual acuity was improved to $20 / 32$.

Pre operation fundus photo and OCT of left eye
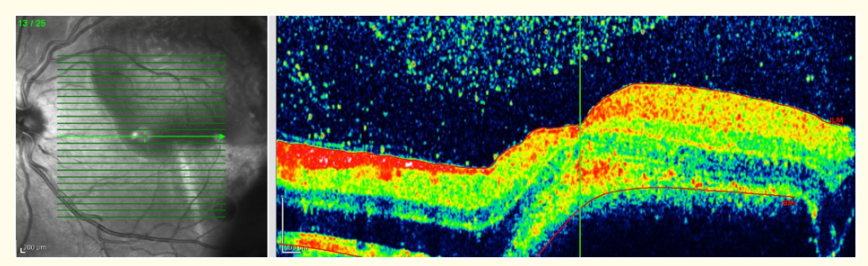

Figure 1

Post operation fudus photo and OCT
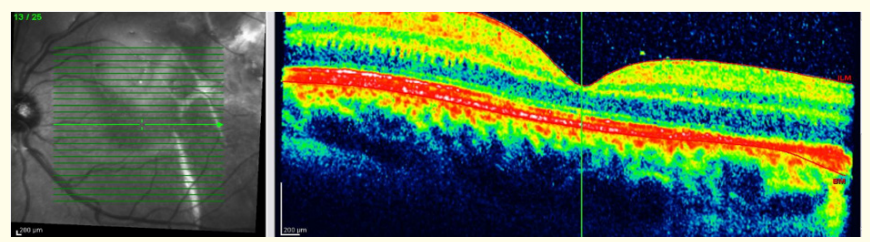

Figure 2

Case 2

A 29 year old male patient with a history of building fall and admission in intensive care unit due to multiple traumas, was referred three weeks after systemic improvement. The visual acuity was finger count of 2 meters and submacular isolated hemorrhage was found. Due to macular involvement, pars plana vitrectomy with submacular hemorrhage sweeping and drainage from a reti- 
notomy one disc diameter superior to the macula was performed. Endolaser around the retinotomy was done and eye was filled with SF6 gas. After 4 weeks, visual acuity was improved to 20/25.

\section{Fundus photo of left eye at ICU}

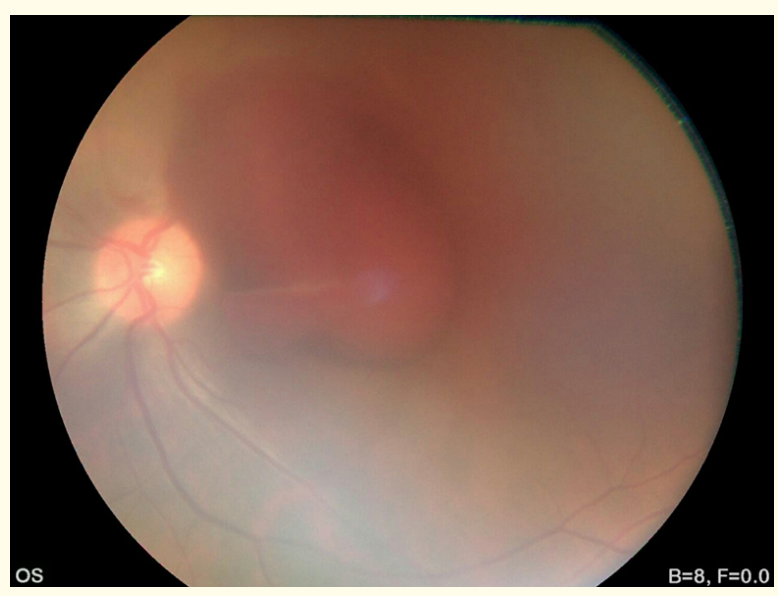

Figure 3

Post operation and sub macular haemorrhage sweeping OCT and fundus photo

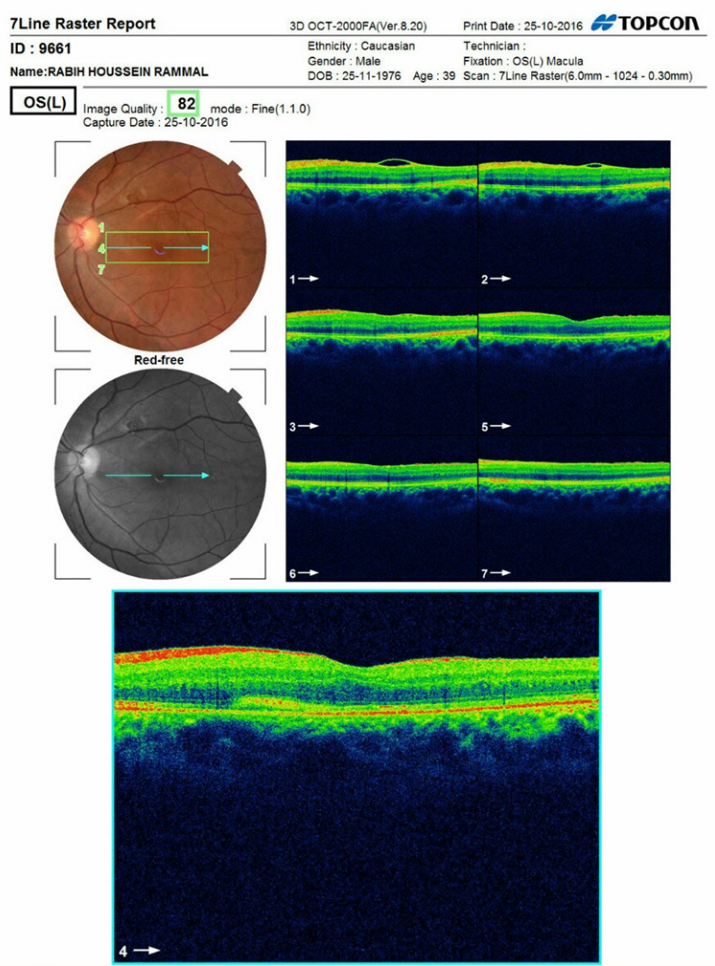

Figure 4
Case 3

A 23 year old male patient with a history of car accident was referred. The visual acuity was hand motion. In fundus exam vitreous hemorrhage, macular hole, choroidal ruptures near the optic disc, submacular hemorrhage and localized retinal detachment around the hole were found. Pars plana vitrectomy with submacular hemorrhage sweeping and guiding the clots through the macular hole without performing internal limiting membrane. Silicone oil was used as the tamponade. After 4 weeks, all the subretinal hemorrhage was removed and visual acuity was improved to 20/100.

Pre operation fundus photo and OCT
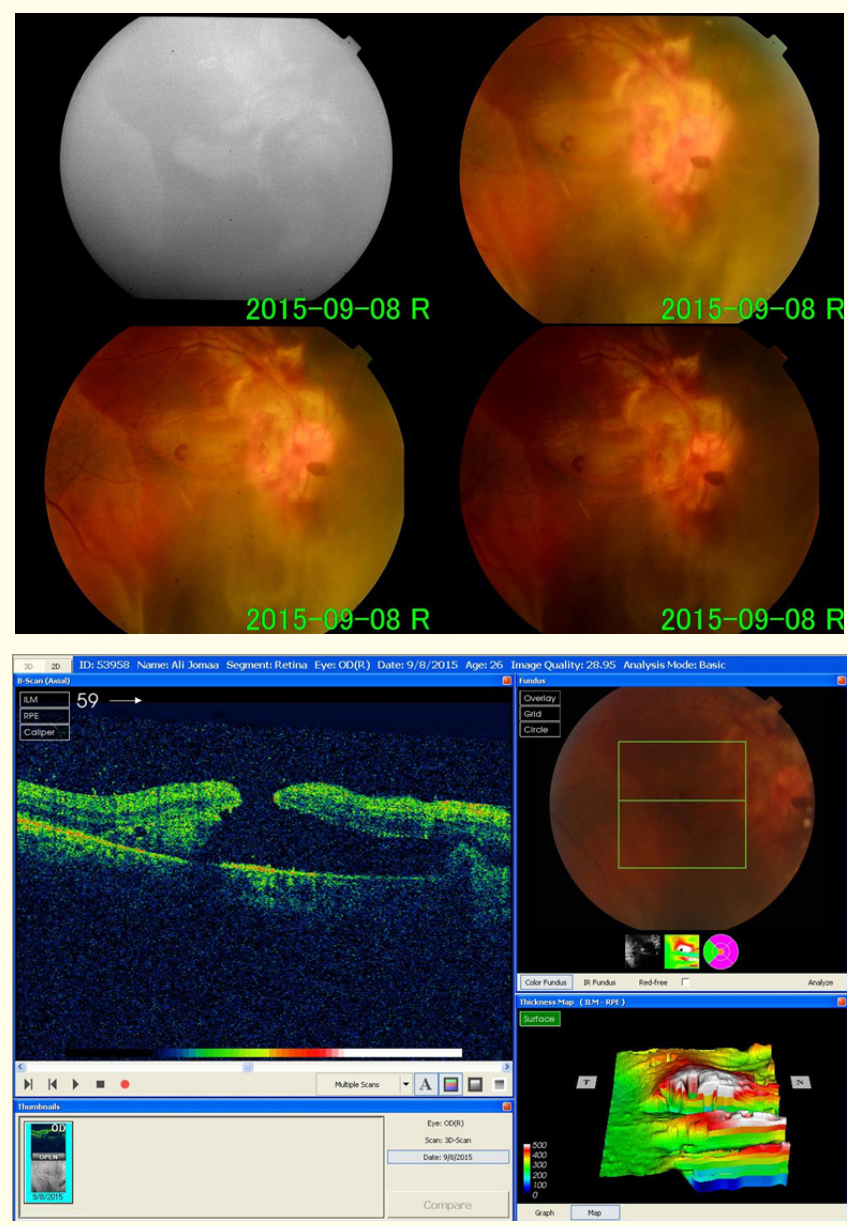

Figure 5

Citation: Mohammad Khalife., et al. "A Novel Surgical Technique of Removal of Sub-Macular Hemorrhage in Post Traumatic Cases". Acta Scientific Ophthalmology 4.4 (2021): 211-215. 
Post operation fundus photo and OCT
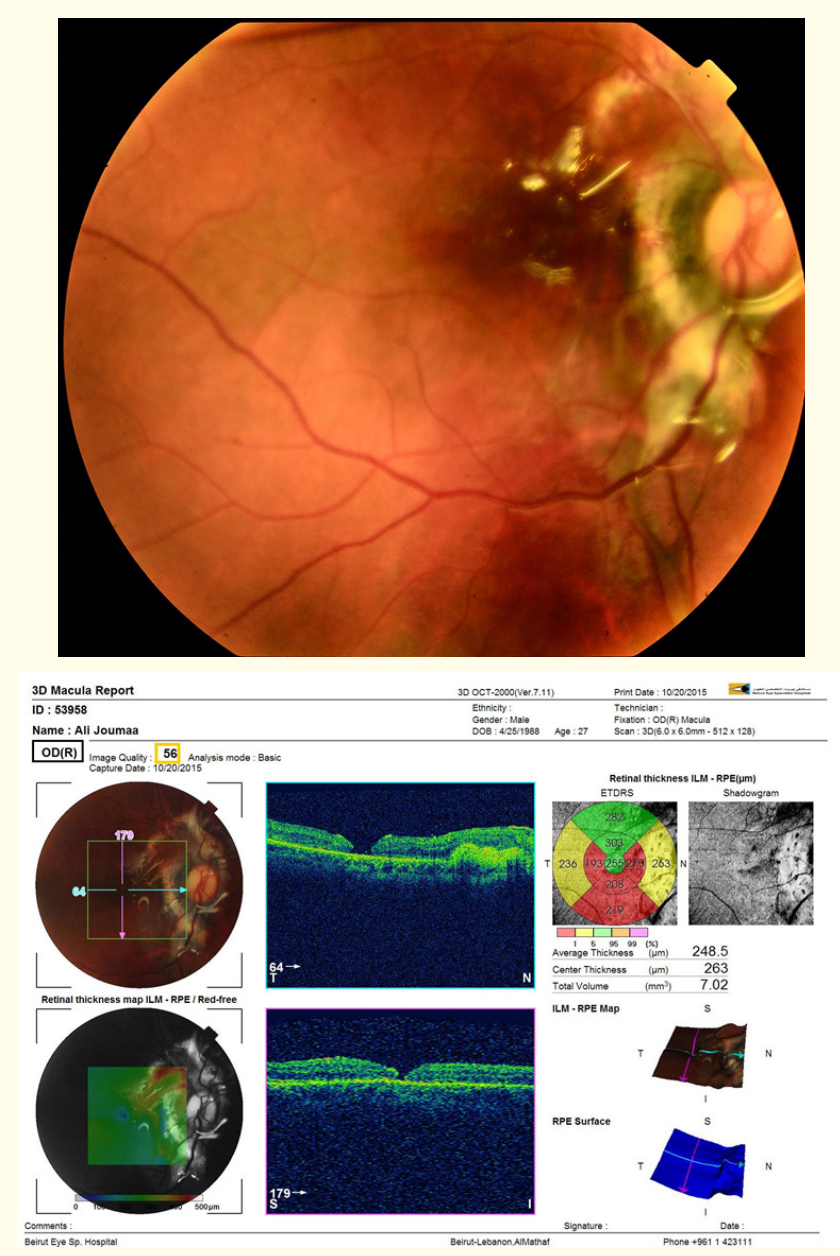

Figure 6

\section{Discussion and Conclusion}

Choroidal rupture may occur in nearly $5 \%$ of ocular blunt trau$\mathrm{ma}$, and if it took place in the macula, visual acuity may not improve better than 20/40 [3]. Although in some case visual loss reversed, SMH affects the photoreceptors and make a permanent visual loss. Iron derived from hemoglobin affects the photoreceptors and has a destructive effect on the outer layers of the retina. SMH form a direct mechanical barrier and deprives the photoreceptors from receiving metabolic support of the choriocapillaries and the RPE [2]. Therefore removing SMH from direct contact to the fovea may save the vision.
Many surgical approaches have been proposed. In most of them a fibrinolytic is used to dissolve the hemorrhage clot and pneumatic displacement from the macula to more peripheral loci and save the fovea [9-11]. In these presented cases, the hemorrhage still remained and may destruct somewhere else in the posterior pole, but not the macula. On the other hand, the most frequent used fibrinolytic is the tPA. It has been shown that tPA is toxic to the retina $[12,13]$. Moreover, in some cases, there is a doubt whether it penetrate enough into the subretinal space or not. Kamei., et al. had shown that intravitreal tPA labeled with fluorescein isothiocyanate did not diffuse across the neurosensory retina to the subretinal space in the rabbit $[11,14]$. Therefore, it is concluded that enough amount of tPA may not enter the subretinal space to liquefy the subretinal blood clot. Therefore, it has been proposed to inject tPA subretinaly during vitrectomy [8].

Due to recent improvements in small gauge vitrectomy in recent years, a surgical approach with adjuvant tPA usage and gas tamponades is preferred instead of previous technics which avoids intraocular surgeries and only included intravitreal injections $[8,14]$.

In our technique, we perform pars plana vitrectomy and used back flush and soft tip head to dislocate the hemorrhage from the subfoveal space to a more temporal area and guide the small clots via a retinotomy. No active or passive aspiration through retinotomy or macular hole were done. We did not use tPA, and our visual results and anatomic changes evaluated by OCT, were shown to be superior to previous techniques, even injections or vitrectomies combined with tPA and expansile gas. We also used expansile gas or silicone oil tamponade, but the main difference is that we drain the subretinal hemorrhage and not just displacing it.

\section{Bibliography}

1. Hochman MA., et al. "Pathophysiology and management of subretinal hemorrhage". Survey of Ophthalmology 42 (1997): 195-213.

2. Glatt $\mathrm{H}$ and Machemer R. "Experimental subretinal hemorrhage in rabbits". American Journal of Ophthalmology 94 (1982): 762-773.

3. Ament CS., et al. "Predictors of visual outcome and choroidal neovascular membrane formation after traumatic choroidal rupture". Archives of Ophthalmology 124 (2006): 957-966. 
4. Ohji M., et al. "Pneumatic displacement of subretinal hemorrhage without tissue plasminogen activator". Archives of Ophthalmology 116 (1998): 1326-1332.

5. Yang P-M., et al. "Pneumatic displacement of a dense submacular hemorrhage with or without tissue plasminogen activator". Chang Gung Medical Journal 28 (2005): 852-859.

6. Holland D and Wiechens B. "Intravitreal r-TPA and gas injection in traumatic submacular hemorrhage". Ophthalmologica 218 (2004): 64-69.

7. Goldman DR., et al. "Traumatic choroidal rupture with submacular hemorrhage treated with pneumatic displacement". Retina 34 (2014): 1258-1260.

8. Doi S., et al. "Successful displacement of a traumatic submacular hemorrhage in a 13-year-old boy treated by vitrectomy, subretinal injection of tissue plasminogen activator and intravitreal air tamponade: a case report". BMC Ophthalmology 15 (2015): 94.

9. Hesse L., et al. "Quantitative effect of intravitreally injected tissue plasminogen activator and gas on subretinal hemorrhage". Retina 20.5 (2000): 500-505.

10. Tsuyama T., et al. "Intravitreal tPA Injection and Pneumatic Displacement for Submacular Hemorrhage in a 10-Year-Old Child". Case Reports in Ophthalmological Medicine (2016): 9809583.

11. Handwerger BA., et al. "Treatment of submacular hemorrhage with low-dose intravitreal tissue plasminogen activator injection and pneumatic displacement". Archives of Ophthalmology 119.1 (2001): 28-32.

12. Chen SN., et al. "Retinal toxicity of intravitreal tissue plasminogen activator: case report and literature review". Ophthalmology 110.4 (2003): 704-708.

13. Hrach CJ., et al. "Retinal toxicity of commercial intravitreal tissue plasminogen activator solution in cat eyes". Archives of Ophthalmology 118.5 (2000): 659-663.
14. Kamei M., et al. "A study of the ability of tissue plasminogen activator to diffuse into the subretinal space after intravitreal injection in rabbits". American Journal of Ophthalmology 128.6 (1999): 739-746.

\section{Assets from publication with us}

- Prompt Acknowledgement after receiving the article

- Thorough Double blinded peer review

- Rapid Publication

- Issue of Publication Certificate

- High visibility of your Published work

Website: www.actascientific.com/

Submit Article: www.actascientific.com/submission.php

Email us: editor@actascientific.com

Contact us: +919182824667 